\title{
Spectrally resolved surface plasmon resonance dispersion using half-ball optics
}

Raphael Dehmel, Jeremy J. Baumberg, Ullrich Steiner, and Bodo D. Wilts

Citation: Appl. Phys. Lett. 111, 201102 (2017); doi: 10.1063/1.4999636

View online: https://doi.org/10.1063/1.4999636

View Table of Contents: http://aip.scitation.org/toc/apl/111/20

Published by the American Institute of Physics

\section{Articles you may be interested in}

Dual-surface flexible $\mathrm{THz}$ Fano metasensor

Applied Physics Letters 111, 201101 (2017); 10.1063/1.5000428

Magnetoplasmonic control of plasmonic vortices

Applied Physics Letters 111, 201104 (2017); 10.1063/1.5001327

High-efficiency and low-loss gallium nitride dielectric metasurfaces for nanophotonics at visible wavelengths Applied Physics Letters 111, 221101 (2017); 10.1063/1.5007007

Focusing light through scattering media by polarization modulation based generalized digital optical phase conjugation

Applied Physics Letters 111, 201108 (2017); 10.1063/1.5005831

Enhanced optical coupling and Raman scattering via microscopic interface engineering

Applied Physics Letters 111, 201105 (2017); 10.1063/1.5003363

Effects of pressure on suspended micromechanical membrane arrays

Applied Physics Letters 111, 201103 (2017); 10.1063/1.5004261

\section{PHYSICS TODAY}

MANAGER'S GUIDE

Accelerate R\&D with

Multiphysics Simulation
READ NOW

PRESENTED BY

マ๐ $\subset$ MSOL 


\title{
Spectrally resolved surface plasmon resonance dispersion using half-ball optics
}

\author{
Raphael Dehmel, ${ }^{1, a)}$ Jeremy J. Baumberg, ${ }^{1}$ Ullrich Steiner, ${ }^{2}$ and Bodo D. Wilts ${ }^{2, b)}$ \\ ${ }^{1}$ Cavendish Laboratory, Department of Physics, University of Cambridge, J.J. Thomson Avenue, \\ Cambridge CB3 OHE, United Kingdom \\ ${ }^{2}$ Adolphe Merkle Institute, University of Fribourg, Chemin des Verdiers 4, 1700 Fribourg, Switzerland
}

(Received 9 August 2017; accepted 20 October 2017; published online 13 November 2017)

In this work, a variant of a Kretschmann-type surface plasmon resonance (SPR) sensor is introduced. $k$-space imaging is combined with half-ball glass optics to facilitate the measurement of SPRs across the visible spectral range. In contrast to current state-of-the-art techniques, which are widely utilized in commercially available systems, the presented method allows single-shotacquisition of the full angular reflection without any moving parts, as well as mapping of the surface plasmon dispersion by scanning across the entire visible wavelength range. Measurements on various thin metallic films demonstrate the sensitivity of the system towards minute changes of the metal surface and its close vicinity. The fast and precise measurement of surface plasmon resonances paves the way for improved detection in applications such as immunoassays or gas-sensors, especially for real-time in situ measurements. Published by AIP Publishing.

https://doi.org/10.1063/1.4999636

Surface plasmon polaritons (SPPs) are surface electromagnetic waves that propagate in a direction parallel to a metal/dielectric interface. These charge density oscillations are confined to an interface between a metal with $\varepsilon_{\mathrm{M}}<0$ and a dielectric with $\varepsilon_{\mathrm{D}}>00^{1,2}$ At such a boundary, SPPs are very sensitive to small changes in $\varepsilon_{\mathrm{D}}$, making them ideal to probe surface chemistries, such as the adsorption of molecules at metal surfaces or the specific binding of biological agents. ${ }^{3}$ The resulting changes in the plasmon resonance give rise to variations in the optical excitation of SPPs, which are typically detected by monitoring the angular reflectance spectrum of light exciting the plasmonic resonances. Surface plasmon resonance (SPR) spectroscopy has been a popular technique in chemical and biological sensing applications for many years due to its high sensitivity and the possibility of in situ and label-free measurements. ${ }^{4-9}$

The excitation of SPPs with light requires the matching of frequency and momentum of photons to the SPP. ${ }^{4}$ This is only possible on a planar surface if photons enter from an excitation medium of $\varepsilon_{\mathrm{E}}>\varepsilon_{\mathrm{D}}$. Experimentally, this is best realised by sandwiching a thin film of a plasmonic metal (commonly silver or gold) between two dielectric materials of $\varepsilon_{\mathrm{D}}$ and $\varepsilon_{\mathrm{E}}$, exciting SPPs from the former and detecting them in the latter (see Fig. S1, supplementary material). The excitation of surface plasmons requires electric field oscillations in the plane of the surface, i.e., p- (or TM-) polarized light. ${ }^{3}$

If the metal film is thicker than the skin-depth (i.e., $>30 \mathrm{~nm}$ ), SPPs obey the dispersion relation

$$
k_{\mathrm{sp}}(\omega)=\frac{\omega}{c}\left(\frac{1}{\varepsilon_{\mathrm{D}}}+\frac{1}{\varepsilon_{\mathrm{M}}}\right)^{-1 / 2} .
$$

\footnotetext{
${ }^{a)}$ Current address: Papierfabrik Louisenthal GmbH, 83701 Gmund a.T., Germany.

b)bodo.wilts@unifr.ch
}

Here, $\varepsilon_{\mathrm{D}}$ and $\varepsilon_{\mathrm{M}}$ are the relative permittivities of the dielectric at the free surface and the metal film, respectively, and $c$ is the speed of light in vacuum. ${ }^{4}$ Since the dielectric constant of the metal is negative in the probed frequency range, changes in $\varepsilon_{\mathrm{D}}$ have a large impact on the dispersion relation, which implies that even trace amounts of adsorbed material cause measurable shifts in the resonance condition. Excitation photons can only couple to the surface plasmon modes when the projections of their wave vectors onto the surface of the metal film match those of the plasmon resonance. Since the in-plane wave vector is conserved, the angular dispersion is

$$
k_{\mathrm{p}}(\omega)=\frac{\omega}{c} \sqrt{\varepsilon_{\mathrm{E}}} \sin \alpha,
$$

with the angle of incidence $\alpha$ (see Fig. S1, supplementary material). This implies that for a given excitation frequency, the resonance condition is fulfilled for a well-defined angle of incidence, which depends on the relation of the dielectric constants

$$
\sin \alpha=\left(\frac{\varepsilon_{\mathrm{E}}}{\varepsilon_{\mathrm{D}}}+\frac{\varepsilon_{\mathrm{E}}}{\varepsilon_{\mathrm{M}}}\right)^{-1 / 2} .
$$

The non-linearity of the sine function enables a high measurement accuracy, particularly at large (grazing) angles of incidence (large $\alpha$ ).

Two configurations are commonly employed to excite SPRs by light. In the Otto configuration, ${ }^{10}$ light is coupled into a glass block, typically a prism, and is totally internally reflected on one of the surfaces under a large angle of incidence, giving rise to an evanescent wave at this glass surface. The probed substrate, often a thin film of metal, is then positioned very close to this surface, but not in direct contact with it, so that the evanescent wave interacts with the electrons in the metal to excite SPPs. In the related Kretschmann 
configuration, ${ }^{2}$ the thin metal film is deposited directly onto the prism. Because of its superior robustness, it is the most commonly used experimental technique. ${ }^{11}$ In a typical measurement, the presence of a dielectric material is detected near the surface of a plasmonic metal film on a prism by varying the incidence angle of a laser light beam reflected from the backside of the film while measuring the specularly reflected light flux. To quantify specific interactions, the surface of the sensor film can be functionalized with a molecule that specifically binds to an analyte of interest. ${ }^{4,12}$ Common sensor metals are gold and silver, but copper, titanium, and chromium have also been used. ${ }^{5,13,14}$

The most adopted method works with a laser/prism configuration and samples the SPRs at a single wavelength by scanning the angular range of interest. The resulting mechanical angular adjustments often impose a trade-off between angular resolution and measurement timescale. Real-time experiments often operate in a fixed-angle mode and extrapolate the resonance angle from the reflected intensity at a slightly lower angle. ${ }^{15,16}$ More importantly, the use of a laser does not allow dispersion measurements, for which a range of wavelengths are required (see however Ref. 17). Dispersion measurements are however particularly interesting for the detection of substances that are not purely dielectric, such as inorganic nanoparticles or fluorescent materials.

Here, we present a SPR measurement method that simultaneously works over a wide spectral range and does not require the angular adjustment of any light beam. It makes use of a standard optical microscope with only minor modifications. The key element is a half-ball glass lens, which replaces the conventional prism (Fig. 1). The metal film is deposited onto a microscope cover slip, which is attached to the flat surface of the half-ball-lens. Using a conventional reflection microscope, light is focussed onto the metal film at

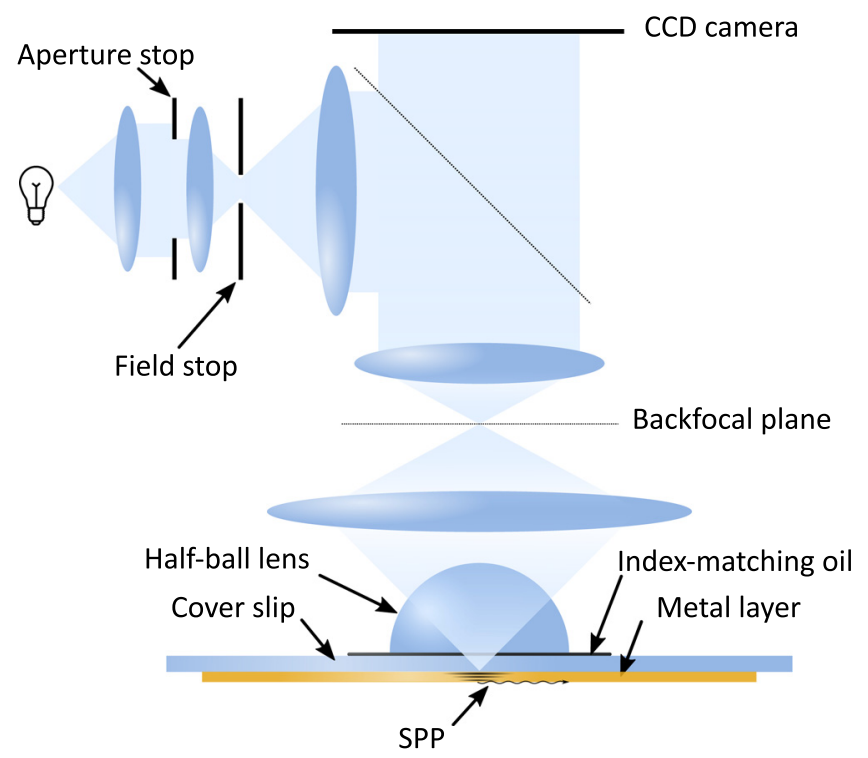

FIG. 1. Schematic of the SPR experimental set-up. The light-cone emitted by a microscope objective passes through a half-ball lens and a cover slip which is metal-coated on its backside. Surface plasmon resonances are excited by photons with polarisation states and angles-of-incidence that match those of SPP modes. The drawing is not to scale (c.f. Fig. S2, supplementary material). the focal point of the sphere. The confocal beam thus enters the glass at normal incidence across the confocal illumination cone, inhibiting refraction and polarization changes at the sphere surface. The same condition holds for the reflected light, which can be detected across the entire numerical aperture of the objective. A light-cone entering the objective from the focal point appears as a circle in the backfocal plane.

The radius of the reflected circle in the backfocal plane correlates with the numerical aperture $\gamma$ of the cone and the focal length $f$ of the objective via $r=\tan \left(\frac{\gamma}{2}\right) \cdot f$.

A Bertrand lens is used to image the far-field scattering pattern of focal point light, by imaging the backfocal plane ( $k$-space imaging or conoscopic imaging ${ }^{18-21}$ ). The detected intensity at any radius $r$ from the optical axis is proportional to the reflectivity of light with an incident angle of $\alpha=\arctan (r / f)$. The intensity at the backfocal plane thus measures the reflectance for all angles of incidence within the numerical aperture of the objective.

A major advantage of this set-up is its wavelengthindependent geometry. With a tunable light source or an appropriate filter set-up, a wide spectral range can be rapidly measured and the wavelength-dependent dispersion of the surface plasmon resonances can be mapped. Furthermore, a standard optical microscope equipped with a Bertrand lens can be used while the absence of moving components makes it robust and precise. We note that high-NA immersion objectives could also be used to excite SPPs in a quite similar fashion. ${ }^{22,23}$

Although the direct deposition of the plasmonic film onto the hemispherical planar part of the half-ball lens would be geometrically ideal, it is experimentally practical to employ a thin glass cover slip instead (Fig. 1). This set-up has several advantages as the externally fixed cover slip supports the halfball-lens in the correct geometry, removing the requirement for a separate alignment mechanism. Furthermore, the use of cover slips significantly simplifies sample preparation and repeated measurements. A refractive index-matching liquid $\left(n_{\mathrm{r}}=1.52\right)$ is deployed between the half-ball lens and the cover slide to prevent interference and interfacial scattering of light (Fig. 1). Note that the normal incidence condition described above is slightly modified, since the metal layer is no longer precisely in the focal plane of the glass hemisphere. Cover-slip thicknesses that are very small compared to the lens radius, as the one employed here, cause only small angular deviations in the backfocal plane, which can be taken into account in the data analysis (see supplementary material). The set-up is used to image the $k$-space of the light reflected by the sample using a long-working-distance $50 \times$ objective. Usually, Köhler illumination images the lamp filament in the backfocal plane, causing strong inhomogeneities in the $k$ space images. This can be avoided by inserting a diffuser into the illumination path or by using a diffuse broadband LED light source (see Materials and Methods in the supplementary material).

An example of a surface plasmon resonance in a $40 \mathrm{~nm}$ thick Au layer is shown in Fig. 2(a). The film was illuminated with unpolarized white light and the radius of the recorded circle corresponds to the maximum angle of incidence of $53.1^{\circ}$, which is determined by the numerical aperture of the objective $(\mathrm{NA}=0.8)$. The refraction caused by 
the finite thickness of the cover slip however slightly compresses the $k$-space image, increasing the cut-off angle to $56^{\circ}$ for a $200 \mu \mathrm{m}$ thick cover slip (see supplementary material, Fig. S4). The unfiltered white light image shows an isotropic ring of increased whitish reflectance close to the critical angle of the BK7 glass of $\alpha \approx 41.1^{\circ}$ (Fig. S7, supplementary material). Below the critical angle, light refracts and interacts with the metal layer according to Snell's law. In the total internal reflection (TIR) region, the whitish-colored ring is surrounded by colored rings formed from an adjacent thin blue ring followed by a broader red ring, indicating the presence of a strong wavelength-dependent resonance [Fig. 2(a)].

To gain quantitative insight into the wavelengthdependent reflection, polarized white light was used and a high-speed liquid-crystal-based tunable color filter was inserted into the optical path. Monochromatic images (spectral half-width $\approx 7 \mathrm{~nm}$ ) were taken across the visible spectrum $(420-680 \mathrm{~nm})$. The reflectance images of the $40 \mathrm{~nm}$ thick gold layer in Figs. 2(b)-2(d) at wavelengths of 470, 570 , and $670 \mathrm{~nm}$, respectively, show clear plasmon resonances which are imaged as dark crescents for p-polarized light [c.f. Fig. S3 (supplementary material) for the polarizationdependence]. The broad, deep plasmon resonance excited by blue light is partially eclipsed at high angles by the limited field of view of the objective [Fig. 2(b)]. This resonance increasingly narrows and shifts to smaller angles with increasing the wavelength [Figs. 2(c) and 2(d)], in qualitative agreement with the white light observation of Fig. 2(a).

With this SPR imaging set-up, SPR sensors consisting of different metal layers ( $\mathrm{Au}, \mathrm{Ag}$, and $\mathrm{Cu}$ ) were examined (Fig. 3). Each measurement takes $\approx 15 \mathrm{~s}$ for a series of monochromatic images covering the entire visible spectrum $(420-680 \mathrm{~nm})$ in $5 \mathrm{~nm}$ steps. Radial intensity profiles were extracted from the $k$-space images and converted to angular reflectance spectra. The conversion is based on a calibration with a known reflection grating and takes into account the finite thickness of the glass cover slips as well as the chromatic aberrations of the glass optics (see supplementary material). In principle, this method allows the mapping of SPP states close to the focus of the half-ball sphere (not shown).

The resulting spectra were compared with analytical calculations and a finite-difference time-domain (FDTD) simulation (see Materials and Methods, Refs. 24 and 25). Both methods, which calculate the angle-dependent reflectance of a Kretschmann-type SPR sensor with a fixed metal layer for p-polarized light, give very similar results (see Fig. S8, supplementary material) and show excellent agreement with the experimental data (Fig. 3). For the $40 \mathrm{~nm}$ thick silver layer [Fig. 3(b)], the reflectance minimum is observed at all wavelengths, shifting towards smaller angles and smaller widths when the wavelength of the incident light increases. These results agree with two well-known SPR properties that (i) silver is the most sensitive SPR sensor metal and that (ii) longwavelength measurements provide the highest resolution, because of the narrow plasmon excitation bandwidth.

It is well known that the metal film thickness is a crucial parameter for the SPR sensor sensitivity. Figure S6 (supplementary material) shows experimental and FDTD spectra of gold layers ranging from 30 to $50 \mathrm{~nm}$, again showing good agreement.

SPR sensors are mainly employed to monitor minute changes in the close vicinity of a metal sensor surface. To probe the ability of our presented set-up to detect small amounts of material, a $2 \mathrm{~nm}$ thick layer of Ag was evaporated onto the $40 \mathrm{~nm}$ thick Au SPR sensor. Figure 4 shows the experimental and FDTD monochromatic $(600 \mathrm{~nm})$ angular scans for a $40 \mathrm{~nm}$ thick Au sensor, uncoated and coated with $2 \mathrm{~nm}$ of Ag. The full reflectance plots, similar to those of Fig. 3, are shown in Fig. S5 (supplementary material). The presence of the thin Ag layer is immediately evident, as (i) the resonance shifts towards shorter wavelengths, (ii) the resonance becomes narrower, and (iii) it varies in intensity. Again, theoretical and experimental data are in very good

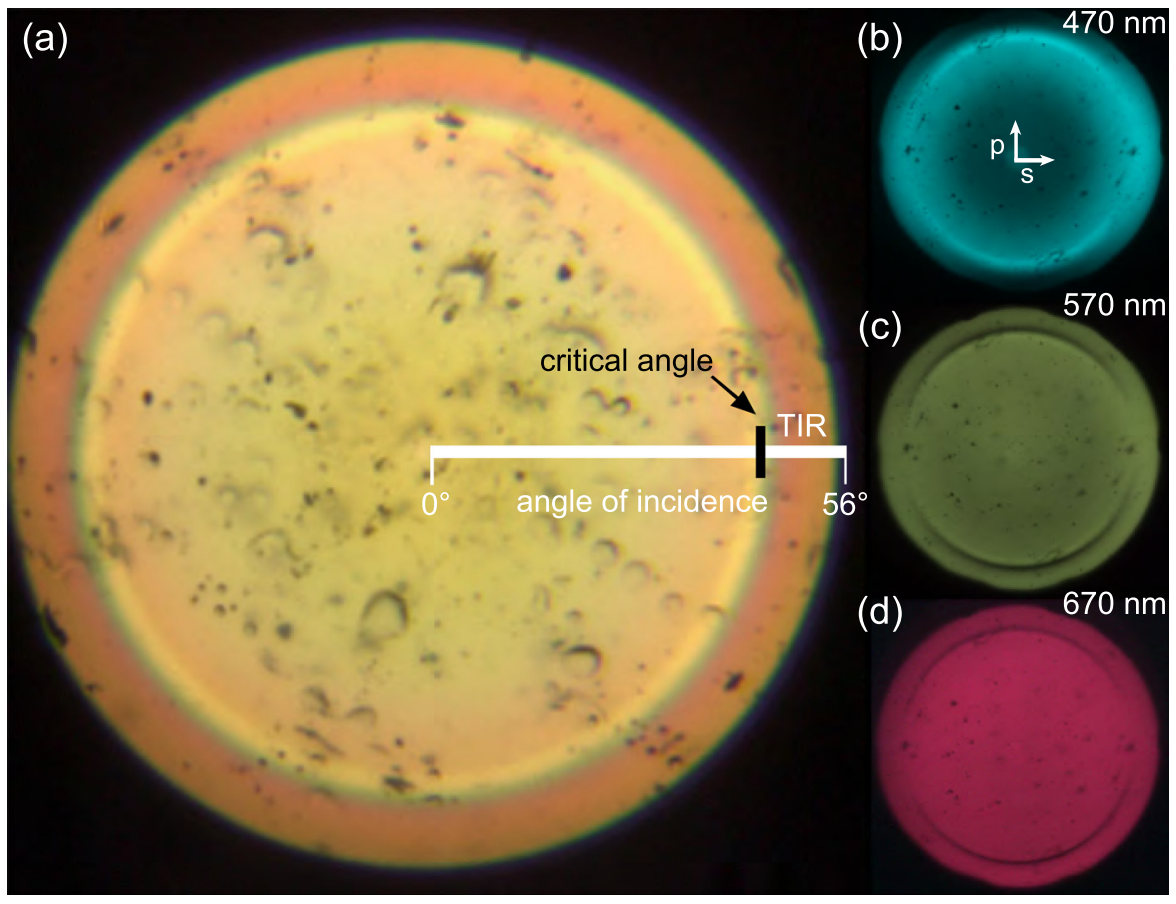

FIG. 2. Backfocal plane images of a $40 \mathrm{~nm}$ thick gold layer illuminated through a glass half-ball. (a) Reflected white-light image of the $k$-space showing a total internal reflection circle. Unpolarized illumination from an incandescent light source with a diffuser. The center of the image corresponds to light scattered normally (angle of incidence $\alpha=0^{\circ}$ ), which radially increases to the maximal scattering angle of $\approx 56^{\circ}$. The critical angle and the total internal reflection (TIR) region are indicated (see text). (b)-(d) Single illumination wavelength images of 470,570 , and $670 \mathrm{~nm}$, respectively, with crescent shaped dark areas of attenuated total internal reflection, indicating resonant surface plasmon excitation. Polarized white LED illumination and a liquid-crystal tunable color-filter were used. The black spots across the images are dirt particles in the imaging optics. 
Experimental

(a)

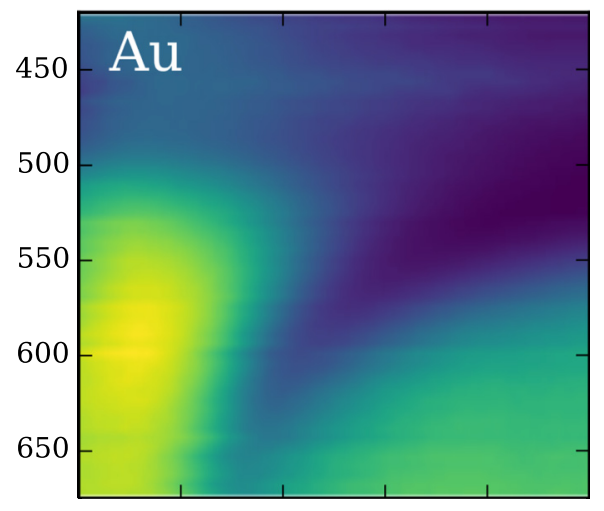

(b)

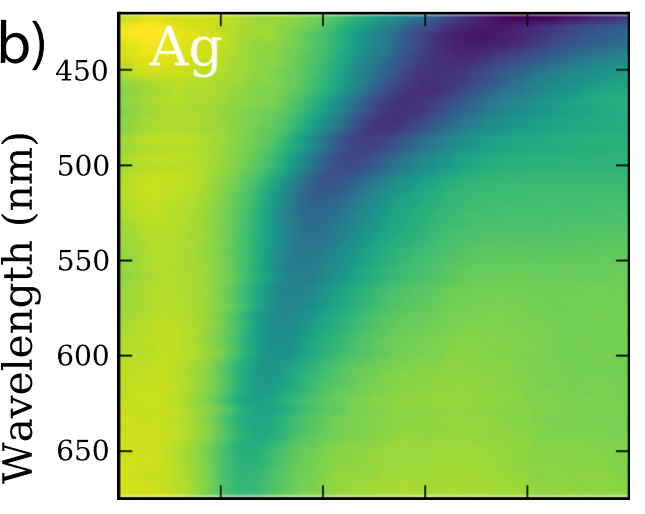

(c)

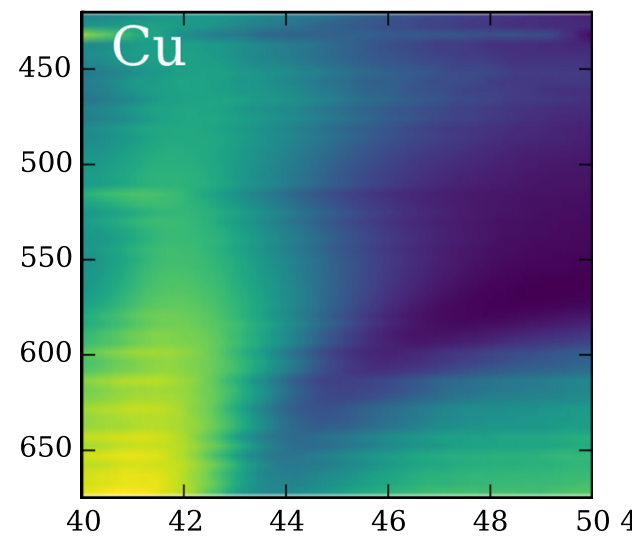

Analytical
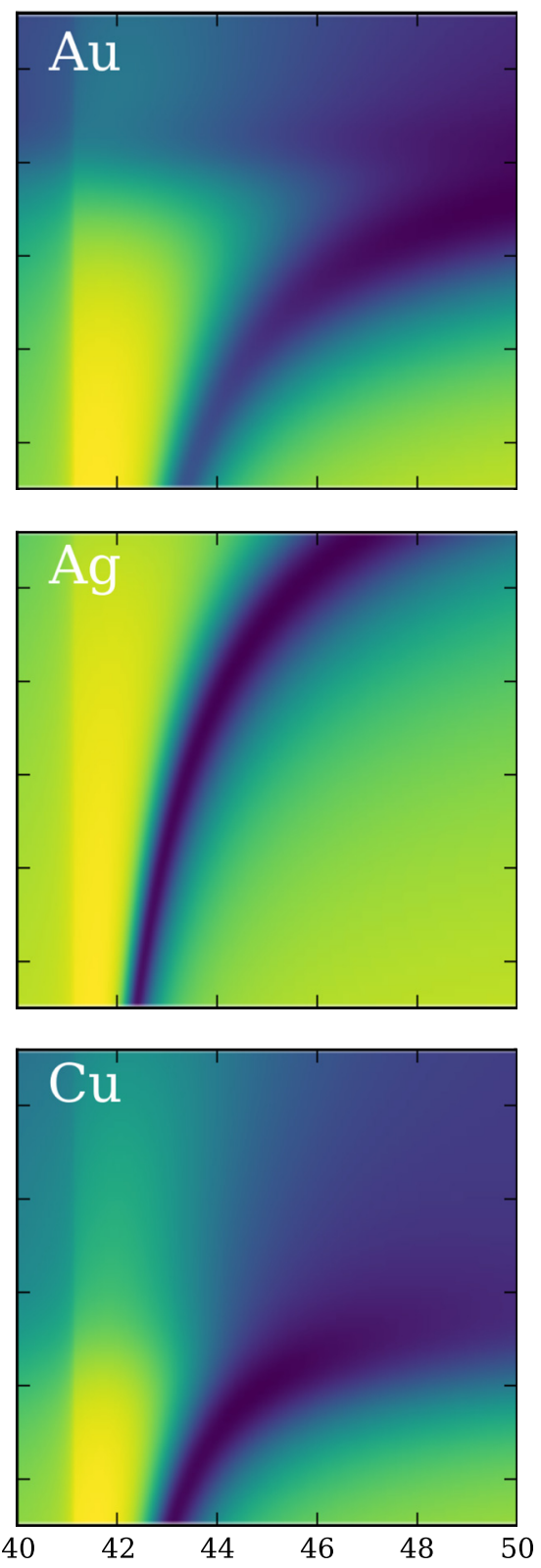

FIG. 3. Surface plasmon resonance dispersion in thin metal layers. Experimentally (left) and theoretically (right) determined surface plasmon dispersion in $40 \mathrm{~nm}$ thick layers of (a) gold, (b) silver, and (c) copper. The cross-over in shading at the critical angle at $\approx 41^{\circ}$ corresponds to the crossover into the TIR region.

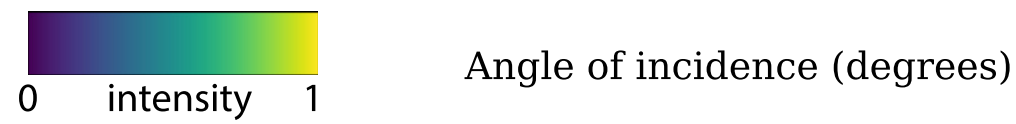

agreement. The smaller amplitude seen in the experimental data is probably caused by local roughness of the metal films and by the limited resolution of the acquisition camera. Despite these limitations, the presence of only $2 \mathrm{~nm}$ thick $\mathrm{Ag}$ layers can be easily detected.

In conclusion, a single-shot SPR method is introduced, which facilitates the rapid characterization of surface layers by exciting surface plasmon resonances over wide angular and spectral ranges. It has the potential to significantly outperform state-of-the-art techniques in two key areas: (i) monochromatic angular reflectance spectra can be recorded without moving parts in single-shot measurements, decreasing the measurement time by orders of magnitude without compromising precision; (ii) the technique is not limited to monochromatic measurements, and a wide range of frequencies can be scanned in an automated way. This adds an extra dimension to the acquired data, which could, for example, facilitate the discrimination between various analytes with similar dielectric constants but different dispersion behaviours and allow full 3D reconstructions of the plasmon dispersion.

Our SPR implementation makes use of standard off-theshelf equipment, which makes the technique very accessible. Its performance could however be further enhanced by using specialized components, e.g., to reach larger scattering angles required for water-based immunoassays used to study biomolecule interactions, which cannot be reached in the current configuration. The angular resolution of our setup depends on the pixel density of the detector in the angular range of interest. A half-ball lens with a higher refractive 

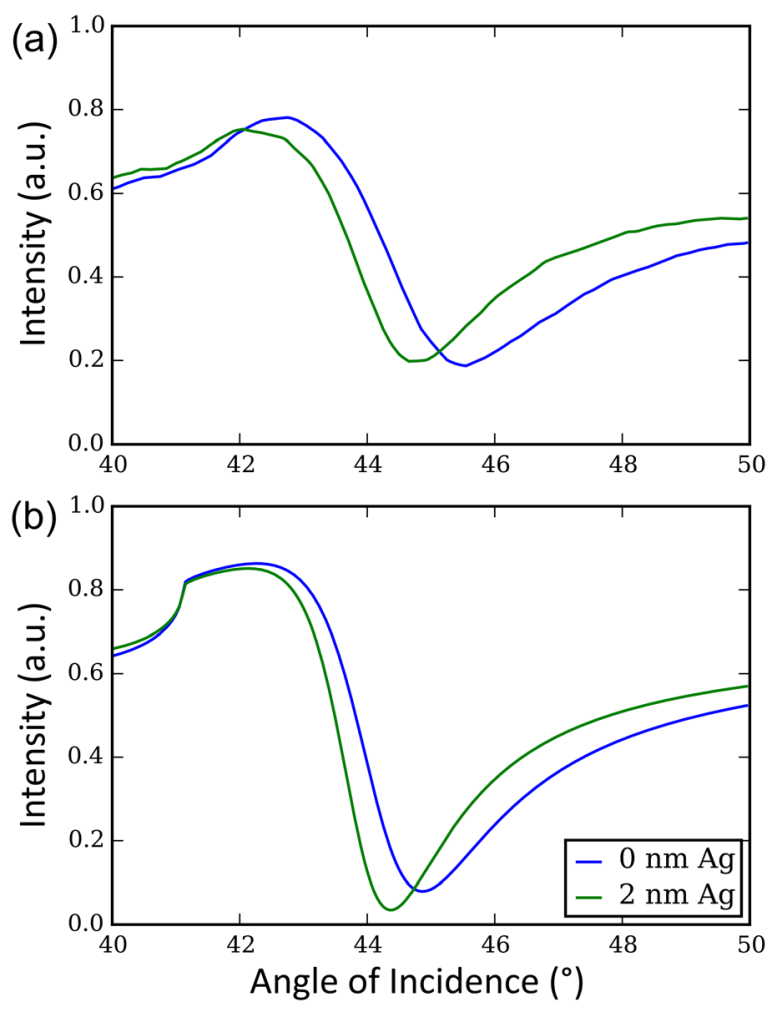

FIG. 4. Resonance shift caused by a $2 \mathrm{~nm}$ thick silver layer on gold. (a) Experimentally and (b) FDTD determined angular reflectance of a $40 \mathrm{~nm}$ thick gold layer with (green line) and without (blue line) a $2 \mathrm{~nm}$ thick silver surface layer illuminated with light of $600 \mathrm{~nm}$ wavelength.

index (e.g., SF6 with $n_{\mathrm{r}}=1.77$ ) shifts this range to lower angles where compression is less significant, while a higherNA objective increases the range of scattering angles that can be measured. Combined with a high pixel-density CCD, this will lead to a much higher angular resolution. A highspeed camera will allow live-tracking of dynamic processes. A high power light source improves the signal to noise ratio and in combination with a better monochromator increases the spectral resolution of the technique.

See supplementary material for the full Materials and Methods and supplementary Figs. S1-S9.
We are much indebted to Karl Christoph Gödel for his invaluable advice on the image analysis algorithms and Stefano Salvatore for sharing initial measurements. This research was partly supported through the National Centre of Competence in Research "Bio-Inspired Materials," the Adolphe Merkle Foundation, the National Center of Competence in Research Bio-Inspired Materials and the Ambizione program of the Swiss National Science Foundation (168223), EPRSC Grant Nos. EP/ G060649/1, EP/L027151/1, and ERC Grant LINASS 320503.

Additional data related to this publication is available at the University of Cambridge data repository (http:// dx.doi.org/10.17863/CAM.14289).

${ }^{1}$ E. Kretschmann and H. Raether, Z. Naturforsch. 23a, 2135 (1968).

${ }^{2}$ E. Kretschmann, Z. Phys. 241, 313 (1971).

${ }^{3}$ A. V. Zayats, I. I. Smolyaninov, and A. A. Maradudin, Phys. Rep. 408, 131 (2005).

${ }^{4}$ B. Liedberg, C. Nylander, and I. Lunström, Sens. Actuators, B 4, 299 (1983).

${ }^{5}$ J. Homola, S. S. Yee, and G. Gauglitz, Sens. Actuators, B 54, 3 (1999).

${ }^{6}$ C.-M. Wu, Z.-C. Jian, S.-F. Joe, and L.-B. Chang, Sens. Actuators, B 92, 133 (2003).

${ }^{7}$ M. Mrksich, G. B. Sigal, and G. M. Whitesides, Langmuir 11, 4383 (1995).

${ }^{8}$ K. V. Gobi, H. Iwasaka, and N. Miura, Biosens. Bioelectron. 22, 1382 (2007).

${ }^{9}$ W. M. Mullett, E. P. Lai, and J. M. Yeung, Methods 22, 77 (2000).

${ }^{10}$ A. Otto and J. B. Swan, Z. Phys. 206, 277 (1967).

${ }^{11}$ R. Karlsson, J. Mol. Recognit. 17, 151 (2004).

${ }^{12}$ D. B. Shuwen Zeng, Chem. Soc. Rev. 43, 3426 (2014).

${ }^{13}$ B. Johnsson, S. Löfås, and G. Lindquist, Anal. Biochem. 198, 268 (1991).

${ }^{14}$ W. A. Weimer and M. J. Dyer, Appl. Phys. Lett. 79, 3164 (2001).

${ }^{15}$ B. P. Nelson, T. E. Grimsrud, M. R. Liles, R. M. Goodman, and R. M. Corn, Anal. Chem. 73, 1 (2001).

${ }^{16}$ J. S. Shumaker-Parry and C. T. Campbell, Anal. Chem. 76, 907 (2004).

${ }^{17}$ T. J. Constant, A. P. Hibbins, A. J. Lethbridge, J. R. Sambles, E. K. Stone, and P. Vukusic, Appl. Phys. Lett. 102, 251107 (2013).

${ }^{18}$ S. Vignolini, E. Moyroud, B. J. Glover, and U. Steiner, J. R. Soc. Interface 10, 20130394 (2013).

${ }^{19}$ P. Vukusic and D. G. Stavenga, J. R. Soc. Interface 6, S133 (2009).

${ }^{20}$ J. Fu, B.-J. Yoon, J. O. Park, and M. Srinivasarao, Interface Focus 7, 20170016 (2017).

${ }^{21}$ J. A. Kurvits, M. Jiang, and R. Zia, J. Opt. Soc. Am. A 32, 2082 (2015).

${ }^{22}$ C. Regan, L. Grave de Peralta, and A. Bernussi, J. Appl. Phys. 111, 073105 (2012).

${ }^{23}$ D. Dominguez, M. Alhusain, N. Alharbi, A. Bernussi, and L. G. de Peralta, Plasmonics 10, 1337 (2015).

${ }^{24} \mathrm{P}$. Yeh, Optical Waves in Layered Media (Wiley-Interscience, 2005).

${ }^{25}$ B. D. Wilts, K. Michielsen, H. A. De Raedt, and D. G. Stavenga, Proc. Natl. Acad. Sci. U.S.A. 111, 4363 (2014). 